\title{
Evaluación de la composición nutricional de alimentos procesados y ultraprocesados de acuerdo al perfil de alimentos de la Organización Panamericana de la Salud, con énfasis en nutrientes críticos
}

\author{
Eliana Meza Miranda ${ }^{1}$, Beatriz Elizabeth Nuñez ${ }^{2}$, Olga Maldonado ${ }^{3}$ \\ ${ }^{1}$ Docente Investigador. Centro Multidisciplinario de Investigaciones Tecnológicas, Universidad Nacional de \\ Asunción. Paraguay \\ ${ }^{2}$ Docente Investigador, Universidad Americana. Paraguay \\ ${ }^{3}$ Facultad de Nutrición, Universidad Autónoma del Paraguay-Pierre Fauchard. Paraguay
}

Cómo referenciar este artículo/ How to reference this article:
Meza Miranda E, Nuñez BE, Maldonado 0 . Evaluación de la composición nutricional de alimentos procesados y ultraprocesados de acuerdo al perfil de alimentos de la organización panamericana de la salud, con énfasis en nutrientes críticos. Mem. Inst. Investig. Cienc. Salud. 2018; 16(1): 54-63

\section{RE S U M E N}

La modernización alimentaria ha llevado a una mayor disponibilidad de alimentos industrializados, ricos en azúcares simples, grasas saturadas y altos en sodio; a ese patrón se le ha considerado la "dieta occidental", concomitante al incremento del sobrepeso y obesidad, y al aumento de la morbilidad y mortalidad por enfermedades crónicodegenerativas. El objetivo de este estudio fue determinar la adecuación de la composición nutricional de alimentos procesados y ultraprocesados de acuerdo al Perfil de Alimentos de la Organización Panamericana de la Salud respecto a los nutrientes críticos según lo declarado en el etiquetado nutricional. Estudio transversal, observacional y descriptivo en el que se analizaron 80 productos: 38 procesados y 42 ultraprocesados. Dentro de la categoría de "Cereales", en comparación con los criterios del Perfil de Nutrientes de la OPS, se ha encontrado que la razón sodio/Kcal supera la proporción 1:1. Además, los azúcares agregados superan el $10 \%$ del total de Kcal de los productos en un $24 \%$. En la categoría de "Bebidas", los azúcares agregados sobrepasan las cantidades sugeridas por la OPS en un $15 \%$ del $10 \%$ permitido. En conclusión se encontraron que en los alimentos procesados muchos ingredientes sobrepasan lo permitido o recomendado por la OPS, por lo que el mejor consejo dietético es basar las dietas en alimentos frescos y mínimamente procesados, y la preparación de platos y comidas frescos.

Palabras clave: alimentos procesados, alimentos ultra procesados, nutrientes críticos.

\section{Evaluation of the nutritional composition of processed and ultraproced foods according to the food profile of the pan american health organization with emphasis on critical nutrients}

\footnotetext{
A B S T R A C T

Modernization of food has led to a greater availability of industrialized foods, rich in simple sugars, saturated fats and high in sodium. This pattern has been considered the "Western diet", which has been associated, concomitant with the increase of overweight and obesity, and to the increase in the morbidity and mortality from chronic-degenerative diseases. The objective of this study was to determine the suitability of the nutritional composition of processed and ultra-processed foods according to the Food Profile of the Pan American Health Organization regarding the critical nutrients as stated in the nutrition labeling. Crosssectional, observational and descriptive study in which a total of 80 products: 38 processed 
and 42 ultraprocessed were analyzed. Within the category of "Cereals", in comparison with the criteria of the Nutrient Profile of PAHO, sodium / Kcal ratio exceeded 1:1 ratio. In addition, the added sugars exceeded $10 \%$ of the total Kcal of the products by $24 \%$. In the category of "Beverages", the added sugars surpassed the amounts suggested by the PAHO by $15 \%$ of the $10 \%$ allowed. In conclusion, many ingredients in the processed foods exceeded what is allowed or recommended by PAHO, so the best dietary advice is to base the diets on fresh and minimally processed foods, and the preparation of dishes and fresh foods.

Keywords: processed foods, ultraprocessed foods, critical nutrients.

\section{INTRODUCCIÓN}

A partir del siglo XIX se han registrado cambios en la alimentación en, prácticamente, todas las poblaciones del mundo, con notables variaciones en los distintos continentes y en sus regiones. Los cambios han sido muy evidentes a partir de la segunda posguerra, en la década de los sesenta del siglo XX, relacionados con los procesos de modernización, particularmente con la industrialización alimentaria. Son procesos crecientes de urbanización y emigración rural-urbana. Se incrementó también la producción y distribución de alimentos industrializados, así como el mayor acceso de las familias a éstos ${ }^{(1)}$.

Los cambios alimentarios han sido impulsados por una economía capitalista y globalizante que domina a la mayoría de las naciones del mundo. Los sistemas alimentarios se rigen, cada vez más, por las exigencias marcadas por los ciclos económicos capitalistas de gran escala. Éstos han impulsado la intensificación de la producción agrícola, la orientación de la política de la oferta y la demanda en torno a determinados alimentos, la concentración del negocio en empresas multinacionales, la ampliación y especialización de la distribución alimentaria a través de unas redes comerciales cada vez más omnipresentes y, en definitiva, la internacionalización de la alimentación. La industria alimentaria abarca no sólo a los países industrializados, sino al resto del mundo, ya que afecta primero a los procesos productivos, algunos de los cuales tienen ahora como objetivo el suministro a gran escala y, más recientemente, afectan al consumo mismo, ya que los productos de la cocina industrial y de la agricultura industrializada juegan un papel determinante en el abastecimiento alimentario de los países en desarrollo(1).

En América Latina, los patrones dietéticos han experimentado profundos cambios en los últimos años. En Brasil, por ejemplo, entre 2002-2003 y 2008-2009, hubo un aumento en la disponibilidad de productos listos para el consumo como resultado del aumento en el consumo de los alimentos ultraprocesados sin distinción de ingreso económico. Durante este período, hubo una disminución significativa en el consumo de alimentos mínimamente procesados y los ingredientes de cocción ${ }^{(2)}$.

La sensación de no tener suficiente tiempo está relacionada con cambios en los patrones de consumo de alimentos, tales como la reducción del tiempo dedicado a la preparación de alimentos en el hogar, un mayor consumo de alimentos listos para el consumo, y la disminución de consumo de alimentos frescos en toda la familia. Este tipo de alimentos, a menudo más baratos que los alimentos frescos, ofrecen comidas altamente energéticas y de sabor agradable y se asocian con dietas menos saludables, lo que contribuye a la aparición de la obesidad y los problemas de salud $\operatorname{crónicos}^{(2)}$.

La modernización alimentaria ha llevado a una mayor disponibilidad de alimentos industrializados, ricos en azúcares simples, grasas saturadas y altos en sodio; a ese patrón se le ha considerado la "dieta occidental", que se ha asociado, en todos los países, concomitante al incremento del sobrepeso y obesidad, al aumento de la morbilidad y mortalidad por enfermedades crónico-degenerativas ${ }^{(3)}$. El ambiente obesogénico es la suma de las influencias que los entornos, las oportunidades o las circunstancias de la vida tienen para promover la obesidad en individuos o la sociedad. Los alimentos industrializados son aquellos procesados por industrias alimentarias, con altos volúmenes de producción y distribución regional o nacional, por lo general son de fácil acceso y se presentan como congelados, enlatados, listos para procesar, cocinar, calentar o consumirse ${ }^{(3)}$.

Ante la pandemia de obesidad y las enfermedades crónicas no transmisibles a las que se asocia, causantes del $65 \%$ de la mortalidad global y del $75 \%$ de las mismas en América Latina, la comunicación para la formación del estilo de vida saludable aumenta su relevancia en la atención primaria de la salud con el propósito de disminuir el consumo de alimentos y bebidas obesogénicas mediante estrategias de comunicación en salud. Ésta es "la 
modificación del comportamiento humano y los factores ambientales relacionados con ese comportamiento que indirectamente promuevan la salud, prevengan enfermedades 0 protejan a los individuos del daño"(4).

Debido a la ausencia de estudios en este campo en nuestro país y por todo lo expuesto anteriormente, se desarrolló el presente trabajo que tuvo como objetivo evaluar la adecuación de los productos procesados y ultraprocesados al Perfil de Nutrientes de la Organización Panamericana de la Salud (OPS) respecto a los nutrientes críticos (sodio, grasas totales, grasas trans y azúcares añadidos).

\section{MATERIALES Y MÉTODOS}

Estudio de tipo observacional descriptivo transversal en el que se analizaron productos alimenticios envasados (procesados y ultraprocesados) disponibles para venta a todo público en cadenas de supermercados en Asunción, frecuentemente consumidos por la población adulta y que contaban con RSPA (Registro Sanitario de Producto Alimenticio). Se excluyeron suplementos nutricionales, infusiones como café, té o yerba mate o sus derivados, azúcar blanca o morena, edulcorantes artificiales, cereales (arroz, trigo y sus derivados), granos (maíz), tubérculos (mandioca, papa o similar), aceites vegetales, frutas, hortalizas, carnes y legumbres.

Se utilizó como base el trabajo "Quantifying associations of the dietary share of ultraprocessed foods with overall diet quality in First Nations peoples in the Canadian provinces of British Columbia, Alberta, Manitoba and Ontario" cuyos autores encontraron un 53,9\% del total de la dieta proveniente de alimentos procesados y ultraprocesados. Se estimó el tamaño de la muestra en 68 productos alimenticios y con una probabilidad de pérdida del $10 \%$ la muestra final mínima fue de 75 productos.

Con la información obtenida de los productos relevados en góndolas de supermercados, se elaboró una tabla descriptiva de la composición nutricional en cuanto a los nutrientes críticos. Se agruparon previamente en grupos de acuerdo a sus características. Los valores de los nutrientes se describieron por 100 gramos de porción comestible o gramos netos. En el caso de los líquidos la composición correspondió a 100 mililitros.

Sodio: medido en $\mathrm{mg} / 100$ gramos o mililitros de cada producto.

Azúcares agregados: medidos en gramos/100 gramos o mililitros de cada producto.

Grasas totales: medidas en gramos/100 gramos o mililitros de cada producto.

Grasas saturadas: medidas en gramos/100 gramos o mililitros de cada producto.

Grasas trans: medidas en gramos/100 gramos o mililitros de cada producto.

Los valores de los nutrientes se compararon al Perfil de nutrientes de la OPS según se detalla a continuación:

Con una cantidad excesiva de sodio, si la razón entre la cantidad de sodio $(\mathrm{mg})$ en cualquier cantidad dada del producto y la energía (kcal) es igual o mayor a 1:1 o sea, mayor o igual a $1 \mathrm{mg}$ de sodio por $1 \mathrm{kcal}$.

Con una cantidad excesiva de azúcares, si en cualquier cantidad dada del producto la cantidad de energía ( $\mathrm{kcal}$ ) proveniente de los azúcares libres (gramos de azúcares libre por $4 \mathrm{kcal}$ ) es igual o mayor a $10 \%$ del total de energía (kcal) o sea, mayor o igual al $10 \%$ del total de energía proveniente de azucares libres.

Con una cantidad excesiva de grasas totales, si en cualquier cantidad dada del producto la cantidad de energía ( $\mathrm{kcal}$ ) proveniente del total de grasas (gramos de grasas totales $\times 9$ $\mathrm{kcal}$ ) es igual o mayor a $30 \%$ del total de energía (kcal)

Con una cantidad excesiva de grasas saturadas, si en cualquier cantidad dada del producto la cantidad de energía ( $\mathrm{kcal}$ ) proveniente de grasas saturadas (gramos de grasas saturadas x $9 \mathrm{kcal}$ ) es igual o mayor a $10 \%$ del total de energía (kcal)

Con una cantidad excesiva de grasas trans, si en cualquier cantidad dada del producto la cantidad de energía ( $\mathrm{kcal}$ ) proveniente de grasas trans (gramos de grasas trans $\times 9 \mathrm{kcal}$ ) es igual o mayor a $1 \%$ del total de energía (kcal).

Los datos se digitalizaron, procesaron y analizaron en una planilla electrónica de Microsoft Office $\AA$ Excel $\AA$ 2016. Los datos cuantitativos se presentaron en promedio y desviación estándar para su posterior análisis y comparación en el Perfil de Nutrientes de la OPS. 


\section{RESULTADOS}

\section{ALIMENTOS PROCESADOS}

De los 80 productos alimenticios analizados, 38 corresponden a alimentos procesados. El análisis se realizó en 100 gramos/mililitros de todos los productos. Dentro de la categoría de "CEREALES", en comparación con los criterios del Perfil de Nutrientes de la OPS, se encontró que la razón sodio/Kcal supera la proporción $1: 1$. Además, los azúcares agregados superan el $10 \%$ del total de Kcal de los productos en un $24 \%$. Sin embargo, la cantidad de grasas totales y de grasas trans no superan lo establecido por la OPS ( $\geq 30 \%$ y $\geq 10 \%$, respectivamente). En la categoría de "BEBIDAS", sólo los azúcares agregados sobrepasan las cantidades sugeridas por la OPS en un $15 \%$ del $10 \%$ permitido. Dentro de la clasificación de "DULCES", también los azúcares agregados fueron los únicos nutrientes críticos que sobrepasan las cantidades permitidas por la OPS en un $60 \%$ del $10 \%$ permitido (Tabla 1).

Tabla 1. Nutrientes críticos en cereales, bebidas y dulces procesados según el perfil de nutrientes de la OPS

\begin{tabular}{|c|c|c|c|c|c|c|}
\hline \multirow{2}{*}{$\begin{array}{l}\text { Grupo de } \\
\text { alimentos } \\
\text { CEREALES }\end{array}$} & \multicolumn{6}{|c|}{ Nutrientes críticos } \\
\hline & Sodio & Azúcares & $\begin{array}{l}\text { Grasas } \\
\text { totales }\end{array}$ & $\begin{array}{l}\text { Grasas } \\
\text { saturadas }\end{array}$ & $\begin{array}{l}\text { Grasas } \\
\text { trans }\end{array}$ & kcal \\
\hline $\begin{array}{l}\text { Tortitas de arroz } \\
\text { integral }\end{array}$ & 1000 & 1 & 2,8 & 0,6 & 0 & 382 \\
\hline $\begin{array}{l}\text { Pan artesano } \\
\text { Pan de sándwich }\end{array}$ & $\begin{array}{l}312,8 \\
327,4\end{array}$ & $\begin{array}{l}21,8 \\
22\end{array}$ & $\begin{array}{l}1,8 \\
6,4\end{array}$ & $\begin{array}{l}0,16 \\
3,4\end{array}$ & $\begin{array}{l}0 \\
0\end{array}$ & $\begin{array}{l}224 \\
258\end{array}$ \\
\hline $\begin{array}{l}\text { Rapiditas } \\
\text { clásicas }\end{array}$ & 348 & 24 & 6,8 & 2,4 & 0 & 270 \\
\hline $\begin{array}{l}\text { Cereales } \\
\text { integrales }\end{array}$ & 250 & 25 & 5 & 0 & 0 & 360 \\
\hline $\begin{array}{l}\text { Tirabuzón } \\
\text { Primavera }\end{array}$ & 35 & 43 & 0,25 & 0 & 0 & 395 \\
\hline $\begin{array}{l}\text { Pasta Mix } \\
\text { Galletitas }\end{array}$ & $\begin{array}{l}14 \\
267\end{array}$ & $\begin{array}{l}4,4 \\
32,5\end{array}$ & $\begin{array}{l}2 \\
16\end{array}$ & $\begin{array}{l}0,5 \\
1,7\end{array}$ & $\begin{array}{l}0 \\
0\end{array}$ & $\begin{array}{l}340 \\
442\end{array}$ \\
\hline $\begin{array}{l}\text { Galletitas } \\
\text { integrales }\end{array}$ & 671 & 9,5 & 17 & 2,4 & 0 & 429 \\
\hline $\begin{array}{l}\text { Promedio } \pm \text { DE } \\
\text { Comparación }\end{array}$ & $358,4 \pm 37,4$ & $20,4 \pm 13,45$ & $6,45 \pm 6,1$ & $1,2 \pm 1,3$ & 0 & $344,4 \pm 77,8$ \\
\hline $\begin{array}{l}\text { con perfil de } \\
\text { OPS }\end{array}$ & 1,04 & 81,4 & 55 & 11,3 & 0 & \\
\hline RESULTADOS & Razón >1 & $\begin{array}{l}24 \% \text { del VCT } \\
(>10 \%)\end{array}$ & $\begin{array}{l}16 \% \text { del VCT } \\
(<30 \%)\end{array}$ & $\begin{array}{l}3,3 \% \text { del } \\
\text { VCT } \\
(<10 \%)\end{array}$ & $\mathbf{0}$ & \\
\hline \multicolumn{7}{|l|}{ BEBIDAS } \\
\hline $\begin{array}{l}\text { Jugo de soja } \\
\text { sabor manzana }\end{array}$ & 17 & 6 & 0,3 & 0 & 0 & 33 \\
\hline Néctar durazno & 11,5 & 5,5 & 0 & 0 & 0 & 44 \\
\hline $\begin{array}{l}\text { Promedio } \\
\text { Comparación }\end{array}$ & $14,25 \pm 1,94$ & $5,75 \pm 0,18$ & $0,15 \pm 0,11$ & 0 & 0 & $38,5 \pm 3,9$ \\
\hline $\begin{array}{l}\text { con el perfil de } \\
\text { OPS }\end{array}$ & 0,4 & 23 & 1,35 & 0 & 0 & \\
\hline RESULTADOS & Razón $<1$ & $\begin{array}{l}15 \% \text { del VCT } \\
(>10 \%)\end{array}$ & $\begin{array}{l}\mathbf{0 , 4 \%} \text { del VCT } \\
(<30 \%)\end{array}$ & $\mathbf{0}$ & $\mathbf{0}$ & \\
\hline \multicolumn{7}{|l|}{ DULCES } \\
\hline $\begin{array}{l}\text { Mermelada } \\
\text { Durazno }\end{array}$ & 20 & 52 & 0 & 0 & 0 & 259 \\
\hline Dulce de batata & 0 & 30,5 & 0 & 0 & 0 & 224 \\
\hline $\begin{array}{l}\text { Dulce de batata } \\
\text { con chocolate }\end{array}$ & 5 & 42,5 & 1 & 0 & 0 & 353 \\
\hline $\begin{array}{l}\text { Promediot DE } \\
\text { Comparación }\end{array}$ & $8,3 \pm 10,4$ & $41,7 \pm 10,8$ & $0,3 \pm 0,6$ & 0 & 0 & $278,7 \pm 66,7$ \\
\hline $\begin{array}{l}\text { con el perfil de } \\
\text { OPS }\end{array}$ & 0,04 & 166,7 & 2,97 & 0 & 0 & \\
\hline RESULTADOS & Razón <1 & $\begin{array}{l}60 \% \text { del VCT } \\
(>10 \%)\end{array}$ & $\begin{array}{l}0,86 \% \text { del } \\
\text { VCT }(<30 \%)\end{array}$ & $\mathbf{0}$ & $\mathbf{0}$ & \\
\hline
\end{tabular}


En cuanto al grupo de ENLATADOS/ENVASADO A, se observó que la razón de sodio/Kcal es mayor a 1, los azúcares agregados sobrepasan el $10 \%$ del total de Kcal de los productos en un $14,5 \%$ y el total de grasas y grasas saturadas se mantienen dentro de las cantidades permitidas por la OPS. Respecto a la categoría de ENLATADOS/ENVASADOS $B$, se ha encontrado que la razón sodio/Kcal es mayor a 1, que las grasas totales superan el $30 \%$ del total de Kcal de los productos y que las grasas saturadas superan el $10 \%$ permitido con un valor de $27,8 \%$. Dentro del grupo de alimentos procesados, en la categoría de LÁCTEOS Y DERIVADOS, los resultados muestran que tanto las grasas totales (55\% del total de Kcal de los productos), las grasas saturadas (39,36\% del total de Kcal de los productos) y grasas trans ( $1 \%$ del total de Kcal de los productos) sobrepasan lo permitido por la OPS ( $\geq 30 \%$, $\geq 10 \%$ y $\geq 1 \%$ respectivamente).

Tabla 2. Nutrientes críticos de enlatados/envasados y lácteos y derivados según el perfil de nutrientes de la OPS

\begin{tabular}{|c|c|c|c|c|c|c|}
\hline \multirow{2}{*}{$\begin{array}{l}\text { Grupo de alimentos } \\
\text { ENLATADOS/ } \\
\text { ENVASADOS A }\end{array}$} & \multicolumn{6}{|c|}{ Nutrientes críticos } \\
\hline & Sodio & Azúcares & $\begin{array}{l}\text { Grasas } \\
\text { totales }\end{array}$ & $\begin{array}{l}\text { Grasas } \\
\text { saturadas }\end{array}$ & $\begin{array}{l}\text { Grasas } \\
\text { trans }\end{array}$ & kcal \\
\hline Choclo enlatado & 235 & 0 & 2,4 & 0,5 & 0 & 78 \\
\hline $\begin{array}{l}\text { Palmitos en rodajas } \\
\text { enlatado }\end{array}$ & 400 & 0 & 0 & 0 & 0 & 38 \\
\hline Arveja enlatada & 570 & 0 & 0,6 & 0 & 0 & 98 \\
\hline Salsa Pomarola & 195 & 4,5 & 0 & 0 & 0 & 40 \\
\hline Mostaza & 1625 & 5 & 0 & 0 & 0 & 75 \\
\hline $\begin{array}{l}\text { Salsa de tomate para } \\
\text { pizza }\end{array}$ & 450 & 3,6 & 0 & 0 & 0 & 33 \\
\hline Promedio \pm DE & $579,2 \pm 530,8$ & $2,2 \pm 2,4$ & $0,5 \pm 0,96$ & $0,08 \pm 0,2$ & 0 & $60,3 \pm 26,8$ \\
\hline $\begin{array}{l}\text { Comparación con el perfil } \\
\text { de OPS }\end{array}$ & 8,8 & 8,72 & 4,5 & & 0 & \\
\hline $\begin{array}{l}\text { RESULTADOS } \\
\text { ENLATADOS/ } \\
\text { ENVASADOS B }\end{array}$ & Razón >1 & $\begin{array}{l}14,5 \% \text { del } \\
\text { VCT } \\
(>10 \%)\end{array}$ & $\begin{array}{l}7,5 \% \text { del } \\
\text { VCT } \\
(<30 \%)\end{array}$ & $\begin{array}{l}1,2 \% \text { del } \\
\text { VCT } \\
(<10 \%)\end{array}$ & 0 & \\
\hline $\begin{array}{l}\text { Atún, maíz y vegetales } \\
\text { envasado }\end{array}$ & 292 & 0 & 8 & 0 & 0 & 254 \\
\hline Atún en sal & 400 & 0 & 2,5 & 0 & 0 & 133 \\
\hline Atún en salsa de tomate & 625 & 0 & 15 & 3 & 0 & 222 \\
\hline Atún en aceite vegetal & 625 & 0 & 17 & 2,5 & 0 & 303 \\
\hline Sardina al tomate & 800 & 0 & 15 & 3 & 0 & 158 \\
\hline Sardinas en aceite vegetal & 647 & 0 & 6 & 2 & 0 & 133 \\
\hline Jamón crudo & 2877,5 & 0 & 9 & 3 & 0 & 203 \\
\hline Carne vacuna enlatada & 1510 & 0 & 27 & 41,4 & 0 & 370 \\
\hline Promedio \pm DE & $972,1 \pm 852,4$ & 0 & $12,4 \pm 7,7$ & $6,9 \pm 14,0$ & 0 & $222,0 \pm 84,3$ \\
\hline $\begin{array}{l}\text { Comparación con el perfil } \\
\text { de OPS }\end{array}$ & 4,4 & 0 & 111,96 & 126,1 & 0 & \\
\hline $\begin{array}{l}\text { RESULTADOS } \\
\text { LÁCTEOS Y DERIVADOS }\end{array}$ & Razón >1 & $\mathbf{0}$ & $\begin{array}{l}50,4 \% \text { del } \\
\text { VCT } \\
(>30 \%)\end{array}$ & $\begin{array}{l}27,8 \% \text { del } \\
\text { VCT } \\
(>10 \%)\end{array}$ & $\mathbf{0}$ & \\
\hline Queso Ibérico & 800 & 0 & 35,5 & 23,8 & 1,3 & 427 \\
\hline $\begin{array}{l}\text { Bebida Láctea de Dulce de } \\
\text { Leche }\end{array}$ & 54,5 & 5 & 0 & 0 & 0 & 45 \\
\hline Chocolatada & 57 & 14,5 & 1,5 & 0,9 & 0,05 & 79 \\
\hline Queso feta Cheddar & 1063 & 0 & 22 & 14 & 0 & 267 \\
\hline Queso reggianito rallado & 1400 & 0 & 32 & 21 & 0 & 470 \\
\hline Queso Katupiry & 1250 & 0 & 50 & 29 & 1 & 610 \\
\hline Flan & 98 & 14 & 4 & 1,4 & 0 & 129 \\
\hline Yogurt con cereales & 85 & 9,8 & 2,5 & 2 & 0 & 111 \\
\hline Queso reggianito rallado & 1400 & 0 & 32 & 21 & 0 & 470 \\
\hline Queso Katupiry & 1250 & 0 & 50 & 29 & 1 & 610 \\
\hline Promedio \pm DE & $742,45 \pm 607,5$ & $4,3 \pm 6,1$ & $23,0 \pm 19,8$ & $14,1 \pm 12,2$ & $0,3 \pm 0,5$ & $\begin{array}{l}322,6 \pm 220, \\
6\end{array}$ \\
\hline
\end{tabular}




\begin{tabular}{|c|c|c|c|c|c|}
\hline $\begin{array}{l}\text { Comparación con el perfil } \\
\text { de OPS }\end{array}$ & 2,1 & 17,2 & 178,23 & 127 & 3,06 \\
\hline RESULTADOS & $\mathbf{R a}$ & $\begin{array}{l}5,33 \% \text { del } \\
\text { VCT } \\
(<10 \%)\end{array}$ & $\begin{array}{l}55 \% \quad \text { del } \\
\text { VCT } \\
(>30 \%)\end{array}$ & $\begin{array}{l}39,36 \% \\
\text { del vCT } \\
(>10 \%)\end{array}$ & $\begin{array}{l}1 \% \text { del } \\
\text { VCT } \\
(>1 \%)\end{array}$ \\
\hline
\end{tabular}

\section{ALIMENTOS ULTRAPROCESADOS}

Dentro de esta clasificación se analizaron un total de 42 productos. En la categoría de CONGELADOS, hemos observado que el contenido de sodio supera la razón 1:1 respecto a las Kcal totales de los productos. El resto de los nutrientes críticos se encuentra dentro de los rangos permitidos por la OPS. Respecto a la categoría de GOLOSINAS, los resultados revelan que la razón de sodio respecto a las Kcal de los productos es mayor a 1 , los azúcares superan ampliamente lo permitido por la OPS en un $42 \%$, las grasas totales en un $38 \%$ y las saturadas en un $21 \%$ de lo permitido. Dentro del grupo de BEBIDAS, se puede observar que el sodio supera la razón 1:1 respecto a las Kcal totales de los productos, además, los azúcares agregados superan el $10 \%$ permitido en un $35 \%$ del total de Kcal totales de los productos. El resto de los nutrientes críticos no se encontraron cantidades significativas en este grupo (Tabla 3 ).

Tabla 3. Congelados, Golosinas y Bebidas ultraprocesados y nutrientes críticos según el Perfil de Nutrientes de la OPS.

\begin{tabular}{|c|c|c|c|c|c|c|}
\hline \multirow{2}{*}{$\begin{array}{l}\text { Grupo de alimentos } \\
\text { CONGELADOS }\end{array}$} & \multicolumn{6}{|c|}{ Nutrientes críticos } \\
\hline & Sodio & Azúcares & $\begin{array}{l}\text { Grasas } \\
\text { totales }\end{array}$ & $\begin{array}{l}\text { Grasas } \\
\text { saturadas }\end{array}$ & $\begin{array}{l}\text { Grasas } \\
\text { trans }\end{array}$ & kcal \\
\hline Nuggets a & 369 & 0 & 1 & 0,5 & 0 & 89 \\
\hline Nuggets b & 369 & 0 & 1 & 0,5 & 0 & 89 \\
\hline $\begin{array}{l}\text { Pizza congelada } \\
\text { vegetariana }\end{array}$ & 573 & 0 & 5,3 & 0 & 0 & 144 \\
\hline Pizza congelada & 642 & 0 & 6,4 & 3 & 0 & 200 \\
\hline $\begin{array}{l}\text { Papas congeladas } \\
\text { noisettes }\end{array}$ & 449 & 0 & 5,3 & 0,7 & 0 & 165 \\
\hline \multirow{4}{*}{$\begin{array}{l}\text { Papas congeladas } \\
\text { Mix primavera } \\
\text { Acelga congelada } \\
\text { Salteado de verduras }\end{array}$} & 389 & 0 & 5 & 2,3 & 0 & 147 \\
\hline & 25 & 0 & 0,4 & 0 & 0 & 64 \\
\hline & 150 & 0 & 0 & 0 & 0 & 24 \\
\hline & 10 & 3,5 & 0,2 & 0,1 & 0 & 32 \\
\hline Promedio \pm DE & $\begin{array}{l}330,7 \\
\pm 224,9\end{array}$ & $0,4 \pm 1,2$ & $2,7 \pm 2,7$ & $0,8 \pm 1,1$ & 0 & $106,0 \pm 61,2$ \\
\hline $\begin{array}{l}\text { Comparación con el } \\
\text { perfil de nutrientes de la } \\
\text { OPS }\end{array}$ & 2,1 & 1,56 & 24,6 & 7,1 & \multicolumn{2}{|l|}{0} \\
\hline RESULTADOS & Razón >1 & $\begin{array}{l}1,5 \% \text { del } \\
\text { VCT } \\
(<10 \%)\end{array}$ & $\begin{array}{l}23 \% \text { del } \\
\text { VCT } \\
(<30 \%)\end{array}$ & $\begin{array}{l}6,7 \% \text { del } \\
\text { VCT } \\
(<10 \%)\end{array}$ & \multicolumn{2}{|l|}{$\mathbf{0}$} \\
\hline \multicolumn{7}{|l|}{ GOLOSINAS } \\
\hline Gomitas dulces & 33 & 63 & 0 & 0 & 0 & 338 \\
\hline Chocolate confitado & 79 & 69 & 19 & 12 & & 447 \\
\hline Bombones & 121 & 42 & 36 & 18 & 0,7 & 553 \\
\hline Chocolate & 109 & 52 & 37 & 20 & 0 & 569 \\
\hline Chocolate oblea & 240 & 50 & 28 & 15,6 & 0 & 523 \\
\hline Barra de cereal a & 192 & 30 & 8,4 & 3,2 & 0 & 336 \\
\hline Barra de cereal b & 153 & 18,5 & 8,6 & 5,7 & 0,14 & 339 \\
\hline \multirow{2}{*}{$\begin{array}{l}\text { Promedio } \pm \text { DE } \\
\text { Comparación con el } \\
\text { perfil de OPS }\end{array}$} & $132,4 \pm 69,5$ & $46,4 \pm 17,8$ & $19,6 \pm 14,6$ & $10,6 \pm 7,8$ & $0,1 \pm 0,3$ & $443,6 \pm 106,2$ \\
\hline & 30 & 185,4 & 176,1 & 95,8 & \multicolumn{2}{|l|}{1,3} \\
\hline RESULTADOS & Razón >1 & $\begin{array}{l}42 \% \text { del } \\
\text { VCT } \\
(>10 \%)\end{array}$ & $\begin{array}{l}38 \% \text { del } \\
\text { VCT } \\
(>30 \%)\end{array}$ & $\begin{array}{l}21 \% \text { del } \\
\text { VCT } \\
(>10 \%)\end{array}$ & \multicolumn{2}{|l|}{$\begin{array}{l}0,3 \text { del } \\
\text { vCT } \\
(<1 \%)\end{array}$} \\
\hline \multicolumn{7}{|l|}{ BEBIDAS } \\
\hline Bebida gaseosa Cola & 4 & 11 & 0 & 0 & 0 & 42 \\
\hline Bebida energizante & 35,2 & 6 & 0 & 0 & 0 & 51,2 \\
\hline Bebida rehidratante & 63 & 6 & 0 & 0 & 0 & 26 \\
\hline Arroz con leche & 52 & 9,5 & 3 & 1 & 0 & 119 \\
\hline Yogurt con cereales & 85 & 9,8 & 2,5 & 2 & 0 & 111 \\
\hline \multirow{2}{*}{$\begin{array}{l}\text { Promedio } \pm \text { DE } \\
\text { Comparación con el } \\
\text { perfil de OPS }\end{array}$} & $47,8 \pm 30,45$ & $8,5 \pm 2,3$ & $1,1 \pm 1,5$ & $0,6 \pm 0,9$ & 0 & $69,8 \pm 42,3$ \\
\hline & 0,7 & 33,8 & 10 & 5,4 & \multicolumn{2}{|l|}{0} \\
\hline RESULTADOS & Razón >1 & $\begin{array}{l}35 \% \text { del } \\
\text { VCT } \\
(>10 \%)\end{array}$ & $\begin{array}{l}14,3 \% \text { del } \\
\text { VCT } \\
(<30 \%) \\
\end{array}$ & $\begin{array}{l}7,7 \% \text { del } \\
\text { VCT } \\
(<10 \%)\end{array}$ & $\mathbf{0}$ & \\
\hline
\end{tabular}


El análisis del grupo de los SNACKS, reveló que el sodio sobrepasa la razón 1:1 respecto a las Kcal totales de los productos, además, que las grasas totales superan el $30 \%$ permitido por la OPS en un $43 \%$ del total de Kcal de los productos. Por otro lado, las grasas saturadas se presentan en su límite permitido en un $10 \%$, lo cual se considera fuera de rango permitido. Dentro del grupo de DESHIDRATADOS E INSTANTÁNEOS, se encontró que el sodio supera la razón $1: 1$ respecto a las Kcal totales de los productos, además, que los azúcares agregados superan el $10 \%$ permitido ampliamente en un $55 \%$ del total de Kcal totales de los productos. Respecto a las grasas totales y saturadas, éstas se mantienen dentro del rango permitido por la OPS (Tabla 3 ).

Tabla 3. Nutrientes críticos según el Perfil de Nutrientes de la OPS en Snacks, deshidratados e instantáneos

\begin{tabular}{|c|c|c|c|c|c|c|}
\hline \multirow{2}{*}{$\begin{array}{l}\text { Grupo de alimentos } \\
\text { SNACKS }\end{array}$} & \multicolumn{2}{|c|}{ Nutrientes críticos } & \multirow[b]{2}{*}{$\begin{array}{l}\text { Grasas } \\
\text { totales }\end{array}$} & \multirow[b]{2}{*}{$\begin{array}{l}\text { Grasas } \\
\text { saturadas }\end{array}$} & \multirow[b]{2}{*}{$\begin{array}{l}\text { Grasas } \\
\text { trans }\end{array}$} & \multirow[b]{2}{*}{ kcal } \\
\hline & Sodio & Azúcares & & & & \\
\hline Snacks sabor jamón y queso & 1216 & 31 & 19 & 7,7 & 0,8 & 453 \\
\hline Papas fritas a & 440 & 0,6 & 32 & 9,1 & 0,2 & 524 \\
\hline Papas fritas Sour Cream & 860 & 2,6 & 32 & 8,8 & 0 & 526 \\
\hline Snack de maíz & 452 & 4,8 & 23 & 2 & 0 & 476 \\
\hline Papas fritas $b$ & 490 & 0 & 28 & 8 & 0,23 & 520 \\
\hline Papas fritas C & 560 & 0 & 20 & 8 & 0 & 456 \\
\hline Jamón frito & 933 & 0 & 40 & 13 & 0 & 846 \\
\hline Snacks de Mandioca & 600 & 0 & 25,2 & 2,8 & 0 & 540 \\
\hline Papas fritas d & 790 & 3,3 & 31 & 3,1 & 0 & 538 \\
\hline Snacks de queso & 620 & 2 & 28 & 1,9 & 0 & 513 \\
\hline Nachos & 1168 & 0 & 27,6 & 4 & 2,4 & 484 \\
\hline Bocaditos de maíz a & 480 & 0 & 20 & 8 & 2,4 & 476 \\
\hline Bocaditos de maíz b & 640 & 0 & 10,4 & 1,6 & 0 & 448 \\
\hline Bocaditos de maíz c & 640 & 0 & 10,4 & 1,6 & 0 & 448 \\
\hline \multirow{3}{*}{$\begin{array}{l}\text { Promedio } \pm \text { DE } \\
\text { Comparación con el perfil de OPS } \\
\text { RESULTADOS }\end{array}$} & $\begin{array}{l}706,4 \pm \\
253,8\end{array}$ & $3,2 \pm 8,2$ & $24,8 \pm 8,3$ & $5,7 \pm 3,7$ & $0,4 \pm 0,9$ & $\begin{array}{l}517,7 \pm 1 \\
00,4\end{array}$ \\
\hline & 1,36 & 12,7 & 222,81 & 51,17 & \multirow{2}{*}{$\begin{array}{l}3,9 \\
0,7 \% \text { del } \\
\text { VCT } \\
(<1 \%)\end{array}$} & \\
\hline & $\begin{array}{l}\text { Razón } \\
>1\end{array}$ & $\begin{array}{l}2,4 \% \text { del } \\
\text { VCT } \\
(<10 \%)\end{array}$ & $\begin{array}{l}43 \% \text { del } \\
\text { VCT } \\
(>30 \%)\end{array}$ & $\begin{array}{l}10 \% \text { del } \\
\text { VCT }\end{array}$ & & \\
\hline \multicolumn{7}{|l|}{$\begin{array}{l}\text { DESHIDRATADOS E } \\
\text { INSTANTÁNEOS }\end{array}$} \\
\hline Jugo en polvo & 200 & 90 & 0 & 0 & 0 & 380 \\
\hline Capuccino soluble & 29,5 & 2,4 & 0,35 & 0,3 & 0 & 22 \\
\hline \multirow{2}{*}{$\begin{array}{l}\text { Pure de papa deshidratado } \\
\text { Sopa de pollo y fideos } \\
\text { deshidratado }\end{array}$} & 1167 & 10 & 12 & 5 & 0 & 385 \\
\hline & 3056 & 0,4 & 0 & 0 & 0 & 272 \\
\hline Alimento achocolatado & 60 & 75 & 2,5 & 0 & 0 & \multirow{6}{*}{$\begin{array}{l}370 \\
347 \\
15 \\
255,9 \pm 1 \\
66,5\end{array}$} \\
\hline Alimento achocolatado Light & 158 & 66 & 0 & 0 & 0 & \\
\hline Caldo de verduras instantáneo & 223 & 1 & 0 & 0 & 0 & \\
\hline Promedio \pm DE & $\begin{array}{l}699,1 \pm \\
1110,5\end{array}$ & $34,97 \pm 40,1$ & $2,1 \pm 4,45$ & $0,8 \pm 1,9$ & 0 & \\
\hline \multirow{2}{*}{$\begin{array}{l}\text { Comparación con el perfil de OPS } \\
\text { RESULTADOS }\end{array}$} & 2,7 & \multirow{2}{*}{$\begin{array}{l}139,9 \\
\mathbf{5 5 \%} \text { del } \\
\text { VCT } \\
(>10 \%)\end{array}$} & 19,1 & \multirow{2}{*}{$\begin{array}{l}6,8 \\
\mathbf{7 , 5} \% \text { del } \\
\text { VCT } \\
(<\mathbf{1 0} \%) \\
\end{array}$} & 0 & \\
\hline & $\begin{array}{l}\text { Razón } \\
>1\end{array}$ & & $\begin{array}{l}16 \% \text { del VCT } \\
(<30 \%)\end{array}$ & & $\mathbf{0}$ & \\
\hline
\end{tabular}

\section{DISCUSIÓN}

La creciente evidencia apoya la tesis de que la comida industrial y el procesamiento es ahora la principal fuerza de conformación de lo que ahora se convertió en un sistema alimentario global, y es un determinante clave de los patrones dietéticos y del estado de salud y bienestar relacionados ${ }^{(5-8)}$. Para investigar esta tesis, un sistema de clasificación de alimentos basado en la extensión y el propósito de los alimentos procesados ha sido desarrollado. Este sistema, identificado como NOVA, incluye un grupo de productos alimenticios principalmente formulado a partir de sustancias refinadas derivadas de alimentos junto con aditivos. Estos se identifican como alimentos procesados y ultraprocesados ${ }^{(5,9,10)}$.

En el presente estudio, se analizaron estos dos grupos de alimentos con la finalidad de evaluar si su contenido nutricional en cuanto a nutrientes críticos se ajusta al Perfil de 
Nutrientes de la OPS. En ambos grupos hemos observado que uno, más de uno o todos ellos se encontraban fuera del rango permitido.

En el grupo de alimentos procesados en 4 de 6 subcategorías, la razón de sodio fue mayor a 1 respecto al total de Kcal del alimento en cuestión, estos fueron los Cereales, Enlatados/envasados a, Enlatados/envasados b y Lácteos y derivados. Esta razón también fue mayor dentro del grupo de alimentos ultraprocesados en todas las subcategorías (Congelados, Golosinas, Bebidas, Snacks y Deshidratados e instantáneos). La presión arterial elevada es una causa importante de enfermedades cardiovasculares (ECV). Hay una clara evidencia de la relación causal entre el aumento de la ingesta de sodio en la dieta y el aumento de la presión arterial (PA) en adultos. Además, existe una evidencia creciente de que la sal juega un papel clave en la regulación de la PA en los niños, y como con los adultos, la PA aumenta con el incremento de la ingesta de sal. Para reducir los riesgos asociados con la ECV, la OMS recomienda que los adultos consuman $<2 \mathrm{~g} /$ día de sodio (equivalente de sal $5 \mathrm{~g} /$ día) ${ }^{(11)}$.

Respecto a los azúcares agregados, en el grupo de alimentos procesados, se ha encontrado que están fuera de rango dentro de las subcategorías de Bebidas, Dulces, Enlatados/envasados a y Lácteos y derivados. En el grupo de alimentos ultraprocesados este nutriente crítico se encontró fuera de rango en las subcategorías de Golosinas, Bebidas y Deshidratados e instantáneos. Existe un extenso cuerpo de evidencia hasta ahora que relaciona el aumento de la ingesta de azúcares agregados o libres con la ganancia de peso, aparición de caries dental, aumento de la presión arterial y de lípidos séricos, Diabetes mellitus tipo 2 y con enfermedad coronaria. En respuesta a esta evidencia, diferentes órganos asesores como la Organización Mundial de la Salud (OMS) recomienda un consumo de azúcares libres o agregados de menos del $10 \%$ de la ingesta de energía, mientras que las directrices del Reino Unido van más allá al aconsejar que la ingesta de azúcares libres no supere el $5 \%$ de la ingesta de energía ${ }^{(12)}$.

En cuanto a las grasas totales y grasas saturadas, se observó que estos nutrientes críticos sobrepasaron la recomendación de la OPS en las subcategorías de Enlatados/envasados b y Lácteos y derivados en el grupo de alimentos procesados. En el grupo de alimentos ultraprocesados, estos nutrientes se encontraron fuera de rango en las subcategorías de Golosinas, Snacks y Deshidratados e instantáneos. Aunque la magnitud del efecto probablemente varía según la ingesta dietética específica y la susceptibilidad, se acepta que las grasas saturadas pueden elevar los niveles sanguíneos del colesterol total (CT). Dado que la mayoría del colesterol en sangre se empaqueta en lipoproteínas de baja densidad, (LDL), las elevaciones en el CT reflejan elevaciones en LDL. Se cree que la LDL eleva el riesgo de ECV y al LDL a menudo se lo conoce como "colesterol malo"(13). En una revisión de 15 estudios con más de 59.000 participantes se encontró que la reducción de la ingesta de grasa saturada condujo a una reducción del $17 \%$ en el riesgo de enfermedades cardiovasculares (incluidas enfermedades cardíacas y accidentes cerebrovasculares) Referencia?. El cambio del tipo de grasa que consumimos, reemplazando las grasas saturadas con grasas poliinsaturadas, parece protegernos mejor, reduciendo nuestro riesgo de problemas cardíacos y vasculares. Cuanto mayor es la disminución de la ingesta de grasa saturada, y cuanto más se reduce el colesterol sérico total, mayor es la protección. Las personas sanas parecen beneficiarse tanto como las personas con mayor riesgo de enfermedad cardíaca o accidente cerebrovascular (personas con presión arterial alta, elevado colesterol sérico o diabetes, por ejemplo), y personas que ya han tenido una enfermedad cardíaca o accidente cerebrovascular ${ }^{(14)}$.

En lo que refiere a las grasas trans dentro del grupo de alimentos procesados en la subcategoría de Lácteos y derivados, se muestra que este nutriente crítico sobrepasa la recomendación del $1 \%$ del total de Kcal. Dentro del grupo de alimentos ultraprocesados fueron los Snacks los alimentos que casi alcanzaron este valor en un 0,7\%. La evidencia demuestra que existe un perfil de nutrición negativo de los alimentos ultraprocesados y documenta su gran impacto negativo en la calidad de los alimentos, particularmente considerando el aumento de la densidad de energía de la dieta, el consumo elevado de grasas trans que va acompañado de un elevado consumo de grasas saturadas, cuyos efectos son similares a las trans, además, de un elevado consumo de azúcares agregados y una ingesta deficiente de fibra en la dieta ${ }^{(15)}$.

Como la categoría de alimentos ultraprocesados ha sido recientemente definida, pocos estudios poblacionales han evaluado la asociación entre su consumo y la morbilidad y la 
mortalidad existen. Sin embargo, estudios realizados en diversos países indican la existencia de asociaciones significativas entre el consumo de alimentos ultraprocesados y síndrome metabólico en adolescentes, dislipidemias en niños y obesidad en todos los grupos de edad ${ }^{(15)}$.

Desde el punto de vista de la salud humana, en la actualidad, la división más destacada de alimentos y bebidas es en términos de su tipo, grado y propósito de procesamiento. Tres principales divisiones están especificadas. Dado esto, el mejor consejo dietético es basar las dietas en alimentos frescos y mínimamente procesados, y la preparación de platos y comidas frescos. Si el objetivo es prevenir enfermedades y mejorar el bienestar, el mejor asesoramiento nutricional sobre productos ultraprocesados, independientemente de sus perfiles nutricionales, es evitarlos $o$ al menos minimizar su consumo. Este enfoque implica una revisión sistemática y directrices dietéticas autorizadas y guías gráficas para lo que respecta a la dieta, nutrición y salud ${ }^{(5)}$.

Pese a que Paraguay cuenta con sus propias Guías Alimentarias, en ellas no se contempla recomendaciones sobre este tipo de alimentos. En una próxima revisión se podría incluir dichas recomendaciones de evitar o disminuir su consumo como se da en el caso de las Guías Dietéticas de Brasil. No obstante, como ya habíamos mencionado anteriormente, recientemente la Dirección de Vigilancia de Enfermedades no Transmisibles ha lanzado una gacetilla haciendo referencia a estos productos ${ }^{(4)}$.

Por otro lado, aunque existen controversias sobre cómo se debe presentar el etiquetado nutricional, esto es el punto más visible de la información de un alimento y constituye uno de los pilares básicos en los que se apoya su comercialización, debe contener toda la información necesaria de su composición nutricional, así como la información sobre los macronutrientes y los nutrientes críticos. También, es una herramienta para que los consumidores tomen decisiones respecto a su alimentación, para conservar la salud y prevenir enfermedades ${ }^{(16)}$.

Como fortaleza de este trabajo, cabe resaltar que es el primero en realizarse en el país con énfasis en alimentos procesados y ultraprocesados, el cual puede utilizarse como antecedente para la realización de otros trabajos en el área y como justificación para realizar consensos sobre el etiquetado nutricional, lo cual ayudará al consumidor a elegir los alimentos que más le convienen en cuanto a salud y nutrición, a través de la educación de la población.

Como limitaciones, se resalta que una gran cantidad de alimentos procesados y ultraprocesados importados tenían su etiquetado en otro idioma o con letras muy pequeñas poco legibles, lo cual dificultó la lectura o elección de dichos productos para su evaluación.

\section{REFERENCIAS BIBLIOGRÁFICAS}

1. Pérez Izquierdo $O$, Nazar Beutelspacher $A$, Salvatierra Izaba B, Elena Pérez-Gil Romo S, Rodríguez L, Mariaca Méndez $R$ et al. Frecuencia del consumo de alimentos industrializados modernos en la dieta habitual de comunidades mayas de Yucatán, México. Estud Soc Rev Investig Científica. 2012;19(39):155-84.

2. D'Avila Helen Freitas KVR. Energy intake from ultra-processed foods among adolescents. Rev paul pediatr. 2017;35(1):54-60.

3. Miranda-Cipriano O, Gómez-Guizado G, Munares-García O A-V. Valores percentilares del contenido de azúcar, grasas y sodio en alimentos industrializados según etiquetado expendidos en lima. Boletín INS. 2014;20(5):104-10.

4. Muñoz-Cano J. La industria de los alimentos y sus campañas de promoción de la salud. Rev Esp Comun En Salud. 2013;4(1):3-16.

5. Monteiro CA. Nutrition and health. The issue is not food, nor nutrients, so much as processing. Public Health Nutr. 2009;12(5):729-31.
6. Ludwig DS. Technology, diet, and the burden of chronic disease. JAMA - J Am Med Assoc. 2011;305(13):1352-3.

7. Stuckler D, McKee M, Ebrahim S, Basu S. Manufacturing epidemics: The role of global producers in increased consumption of unhealthy commodities including processed foods, alcohol, and tobacco. PLoS Med. 2012;9(6): 10 .

8. Moodie R, Stuckler D, Monteiro C, Sheron N, Neal B, Thamarangsi $T$, et al. Profits and pandemics : prevention of harmful eff ects of tobacco, alcohol, and ultra-processed food and drink. Lancet [Internet]. 2013;381(9867):670-9. Available from: http://dx.doi.org/10.1016/S01406736(12)62089-3.

9. Moubarac JC, Parra DC, Cannon G MC. Food Classification Systems Based on Food Processing: Significance and Implications for Policies and Actions: A Systematic Literature Review and Assessment. Curr Obes Rep. 2014;3(2):256-72.

10. Monteiro CA, Cannon G, Moubarac JC, Levy RB, Louzada MLC, Jaime PC. The un 
Decade of Nutrition, the NOVA food classification and the trouble with ultraprocessing. Public Health Nutr. 2017;1-13.

11. O'Halloran SA, Grimes CA, Lacy KE, Nowson CA, Campbell KJ. Dietary sources and sodium intake in a sample of Australian preschool children. BMJ Open. 2016;6(2):1-9.

12. Latasa P, Louzada MLDC, Martinez Steele E, Monteiro CA. Added sugars and ultraprocessed foods in Spanish households (1990-2010). Eur J Clin Nutr [Internet]. 2017; Available from: http://www.nature.com/articles/s41430017-0039-0.

13. Long $M$, Tao $S$, Vega $D$, Jiang $T$, Wen $Q$, Sophia L. The Evidence for Saturated Fat and for Sugar Related to Coronary Heart Disease. Prog Cardiovasc Dis. 2016;8(5):444-54.
14. Hooper L, Martin N, Abdelhamid A. G DS. Reduction in saturated fat intake for cardiovascular disease (Review). Cochrane Database Syst Rev. 2015;(6):1-150.

15. Louzada ML da C, Martins APB, Canella DS, Baraldi LG, Levy RB, Claro RM, et al. Ultraprocessed foods and the nutritional dietary profile in Brazil. Rev Saude Publica. 2015;4.

16. Liliana Amparo $\mathrm{L}$ SLR. Etiquetado nutricional, una mirada desde los consumidores de alimentos / Nutritional labeling, from a consumers viewpoint. Perspect En Nutr Humana. 2014;16(2):145-56. 